\title{
Posicionamiento en buscadores para la difusión digital de artículos científicos
}

\section{Search engine optimization for digital dissemination of scientific articles}

\author{
Sebastián Iglesias-Osores ${ }^{1, a}$
}

\section{Sr. Editor}

Con el advenimiento del Internet y la red mundial "www" (del inglés, World Wide Web), la "búsqueda" de información se ha convertido en un importante pilar en la globalización, haciéndola competitiva y comercial. En este contexto las bibliotecas electrónicas y redactores científicos juegan un rol fundamental dentro de este mercado. Otras partes interesadas incluyen, entre otros, editores, integradores de contenido en línea y optimizadores de búsqueda en Internet (1). El posicionamiento en buscadores o SEO (del inglés, search engine optimization) es el proceso de mejorar la posición de un contenido web para que la página aparezca en los resultados de búsqueda de los principales buscadores de Internet. Todos los buscadores tienen una forma única de clasificar la importancia de un sitio web; algunos se centran en el contenido, mientras que otros revisan los metadatos para identificar quién y qué es el negocio de un sitio web ${ }^{(2)}$. El objetivo de esta carta es dar a conocer herramientas informáticas para que los trabajos de los jóvenes investigadores tengan mayor visibilidad en la era digital.

La web invisible académica consta de todas las bases de datos y colecciones relevantes para la academia, pero que los buscadores de Internet de propósito general no pueden buscar. La indexación de esta parte de la web invisible es fundamental para los buscadores científicos de internet en la que muchos artículos de investigación están incluidos ${ }^{(3)}$. Las publicaciones científicas difundidas en la web, se pueden acoger a los criterios del SEO para obtener mejores calificaciones para aparecer en los primeros lugares de las listas de búsqueda, que da más ventaja al momento de ser citado.

Es importante observar de cerca a los lectores y a su comportamiento de uso, para utilizar una suerte de optimización académica que implica la creación, publicación y modificación de literatura académica, de tal manera que facilita a los buscadores académicos de Internet rastrearlos e indexarlos ${ }^{(4)}$. Asimismo el agregado de metadatos en el título y el resumen es especialmente útil, porque permiten a los buscadores atribuir valores semánticos al contenido de la página web y clasificarlos adecuadamente en los resultados de búsqueda logrando hasta un $90 \%$ de indexación ${ }^{(5)}$.

Los artículos científicos deben contener las palabras clave registradas en los Descriptores en Ciencias de la Salud (DeCS) (http://decs.bvs.br/), de la Biblioteca Virtual en Salud del Centro Latinoamericano y del Caribe de Información de Ciencias de la Salud (BIREME), y en inglés el Medical Subject Headings (MeSH terms) (https://meshb.nlm.nih.gov). También es necesario introducir, en el texto del título y el cuerpo del documento, palabras relacionadas al tema tratado que se encuentren en las primeras posiciones del Planificador de Palabras de Google (https://ads.google.com/aw/keywordplanner/home) y Google Trends (https://trends.google.es/trends/); esto junto a una buena redacción y aplicación de las normas de redacción científica ayudará a que el artículo tenga un mejor visibilidad y posicionamiento en los buscadores de Internet.

Estas herramientas también se deberían aplicar al momento de la construcción de los sitios web y repositorios de las revistas para que los artículos ahí publicados tengan mayor visibilidad. La divulgación y la diseminación de la ciencia son los objetivos de toda nueva investigación, que muchas veces no se cumple a cabalidad. Con el uso de estas herramientas estaremos ayudando a que los trabajos se divulgan con mayor amplitud.

\footnotetext{
1. Facultad de Ciencias Biológicas, Universidad Nacional Pedro Ruiz Gallo, Lambayeque, Perú.

a. Biólogo, Microbiólogo.
}

Correspondencia: Sebastián Iglesias-Osores

correo: siglesias@unprg.edu.pe https://doi.org/10.37065/rem.v5i3.370 
Se concluye que, el posicionamiento en buscadores o SEO, mediante la aplicación de estrategias como el ingreso de metadatos y uso de descriptores dentro de nuestros escritos científicos, son de utilidad para dar más visibilidad a las publicaciones científicas y académicas. Su importancia es, en particular, en jóvenes científicos, quienes aún están iniciándose en el mundo académico y por lo tanto no tienen un factor de impacto relevante en la comunidad científica y en los buscadores.

\section{REFERENCIAS BIBLIOGRÁFICAS}

1. Summann F, Lossau N. Search engine technology and digital libraries: Moving from theory to practice. D-Lib Mag. 2004;10(9). doi:10.1045/september2004-lossau

2. Lawrence S, Giles CL. Searching the Web: general and scientific information access. First IEEE/POPOV Work Internet Technol Serv Proc (Cat No99EX391). 1999;37(1):116-122. doi:10.1109/INTS.1999.874002

3. Lewandowski D, Mayr P. Exploring the academic invisible web. Lossau N, ed. Libr Hi Tech. 2006;24(4):529-539. doi:10.1108/07378830610715392

4. Beel JJ, Gipp B, Wilde E. Academic Search Engine Optimization (ASEO): Optimizing Scholarly Literature for Google Scholar \& Co. J Sch Publ. 2010;41(2):176-190. doi:10.3138/jsp.41.2.176.

5. Onaifo $D$, Rasmussen $D$. Increasing libraries' content findability on the web with search engine optimization. Libr Hi Tech. 2013;31(1):87-108. doi:10.1108/07378831311303958

Revisión de pares: Recibido: 19/09/2019 Aceptado: 04/10/2019 